\title{
In situ Scanning Transmission Electron Microscopy with Atomic Resolution under Atmospheric Pressures
}

\author{
Sheng Dai ${ }^{1}$, Shuyi Zhang ${ }^{1}$, George. W. Graham ${ }^{1}$, and Xiaoqing Pan ${ }^{1,2}$ \\ 1. Department of Chemical Engineering and Materials Science, University of California - Irvine, Irvine, \\ CA, USA \\ 2. Department of Physics and Astronomy, University of California - Irvine, Irvine, CA, USA
}

In situ electron microscopy study under gaseous environment has attracted attention not only from basic scientific research but also for important industrial applications. Although differential pumped environmental TEM (ETEM) is a well-established platform to investigate the dynamic gas-solid interaction, some constraints still exist at the current stage: (1) The maximum gas pressure allowed in ETEM is no more than $1 / 100$ of the atmospheric pressure, which is not favorable for building a bridge between the in situ results and real applications. (2) It is difficult to obtain atomic-resolution high angle annular dark-field scanning transmission electron microscopy (HAADF-STEM) images since no probe corrector is available in commercialized ETEMs while ADF-STEM imaging is limited to the low-angle regime due to the post-specimen differential pumping apertures. Recently, it has become possible to overcome these limitations through the use of a MEMS-based, electron-transparent windowed gas cell. Using this instrumentation, the gaseous environment is normally sealed between two silicon nitride windows, reaching the pressure of 760 Torr $(1.0 \mathrm{~atm})$ under static gas conditions or with low flow rates [1-4]. The gas cell holder can be safely inserted in any state of art TEMs without any modification to the column and vacuum system, thus removing the limitation of HAADF-STEM imaging encountered in differentially pumped ETEMs. In this talk, we illustrate the advantages of the windowed gas cell as applied to our in situ study of two important systems: (1) the CO-induced Pt nanoparticle surface reconstruction at saturation coverage (2) the ordering and $\mathrm{Pt}$ surface enrichment in supported $\mathrm{Pt}_{3} \mathrm{Co}$ nanoparticles.

The first example is an observation of structural rearrangement of supported Pt nanoparticle surfaces induced by the adsorption of $\mathrm{CO}$ at saturation coverage and elevated temperature [1], as shown in Figure 1. It is well-known that different geometric configurations of surface atoms on supported metal nanoparticles have different catalytic reactivity and that the adsorption of reactive species can cause reconstruction of metal surfaces. Thus, characterizing metallic surface structures under reaction conditions at atomic scale is critical for understanding reactivity. Here, we observed the truncated octahedron shape adopted by bare Pt nanoparticles undergoes a reversible, facet-specific reconstruction due to $\mathrm{CO}$ adsorption, where flat (100) facets roughen into vicinal stepped high Miller index facets, while flat (111) facets remain intact. It is noticeable that high partial pressure of CO (>20 Torr) allowed in the gas cell, ensuring the saturation $\mathrm{CO}$ coverage, triggered the surface reconstruction of $\mathrm{Pt}$ nanoparticles. The in-situ electron microscopy evidence shows excellent agreement quantitatively with the result of density functional theory (DFT)-based calculation, providing a clear insight for CO-induced reconstruction of (100) sites.

Another example is the in situ STEM study of surface evolution in $\mathrm{Pt}_{3}$ Co nanoparticles [2-3], which is important for the oxygen reduction reaction (ORR) in fuel cell catalysts. To better understand the formation process of the Pt shell, which is crucial for enhancing ORR activity, we performed in situ experiment on carbon-supported $\mathrm{Pt}_{3} \mathrm{Co}$ nanoparticles under atmospheric oxygen pressure. The initially disordered $\mathrm{Pt}_{3} \mathrm{Co}$ nanoparticles were found to transform to an ordered intermetallic phase after high 
temperature $\left(720{ }^{\circ} \mathrm{C}\right)$ annealing in 760 Torr of $\mathrm{O}_{2}$, then followed by layer-by-layer Pt shell growth on (100) surfaces via Ostwald ripening at low temperature $\left(300^{\circ} \mathrm{C}\right)$, as shown in Figure 2 . The intermetallic core and the $\mathrm{Pt}$ shell were revealed directly through differences in atomic number $(\mathrm{Z})$ contrast of HAADF-STEM images. Further intensity analysis, combined with STEM image simulation confirms the composition of the newly grown Pt layers and reflects the layer-by-layer growing process quantitatively. This novel oxygen-driven core-shell formation may thus pave a way for designing and tailoring the structure and performance of Pt-Co ORR catalyst.

\section{References:}

[1] T Avanesian, et al. J. Am. Chem. Soc. 139 (2017), p. 4551.

[2] S Dai, et al, Nat. Commun. 8 (2017), p. 204.

[3] S Dai, et al. Nano Lett. 17 (2017), p. 4683.

[4] S Dai, et al. ACS Catal. 7 (2017) p. 1579.
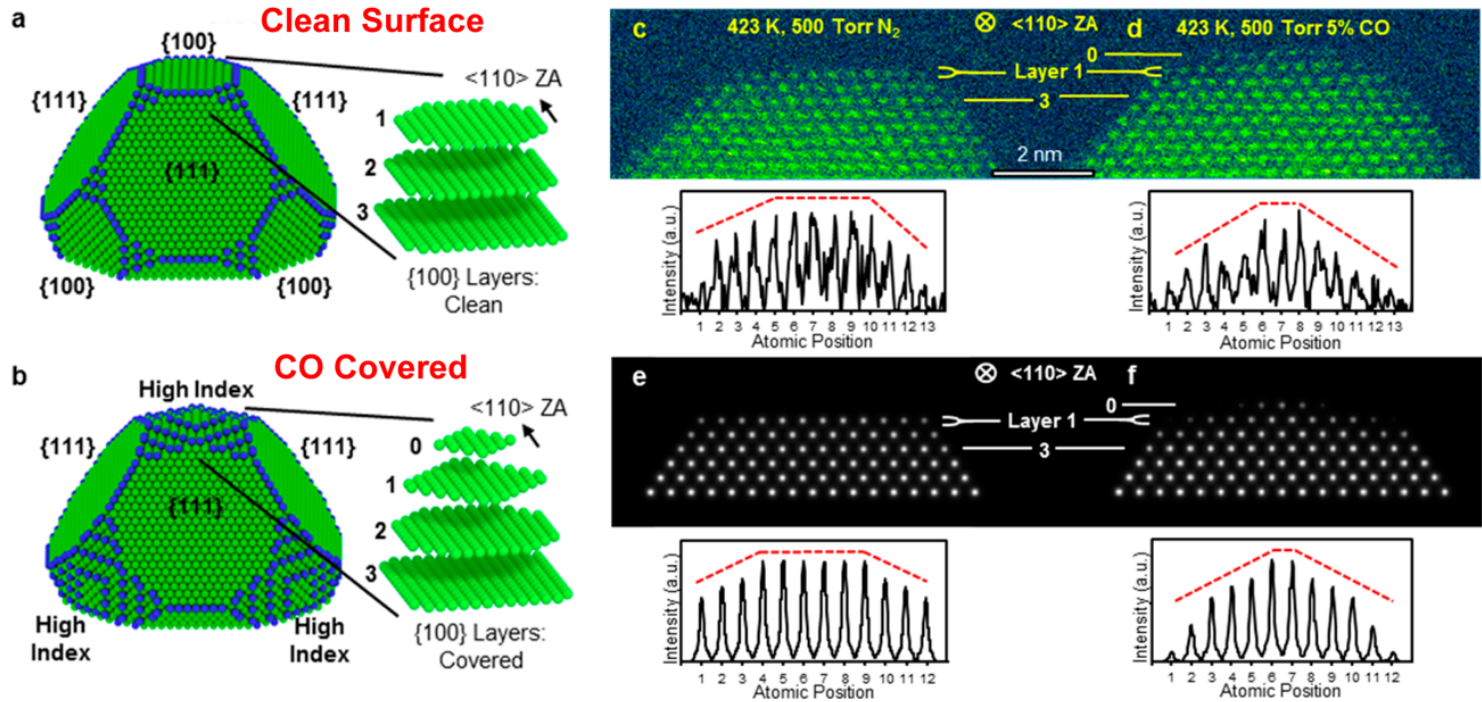

Figure 1. Visual comparison of DFT-calculated (a-b) and in situ STEM measurements (c-f) of facetspecific CO-induced Pt nanoparticle surface reconstruction. Intensity analysis of layer 1 was presented.
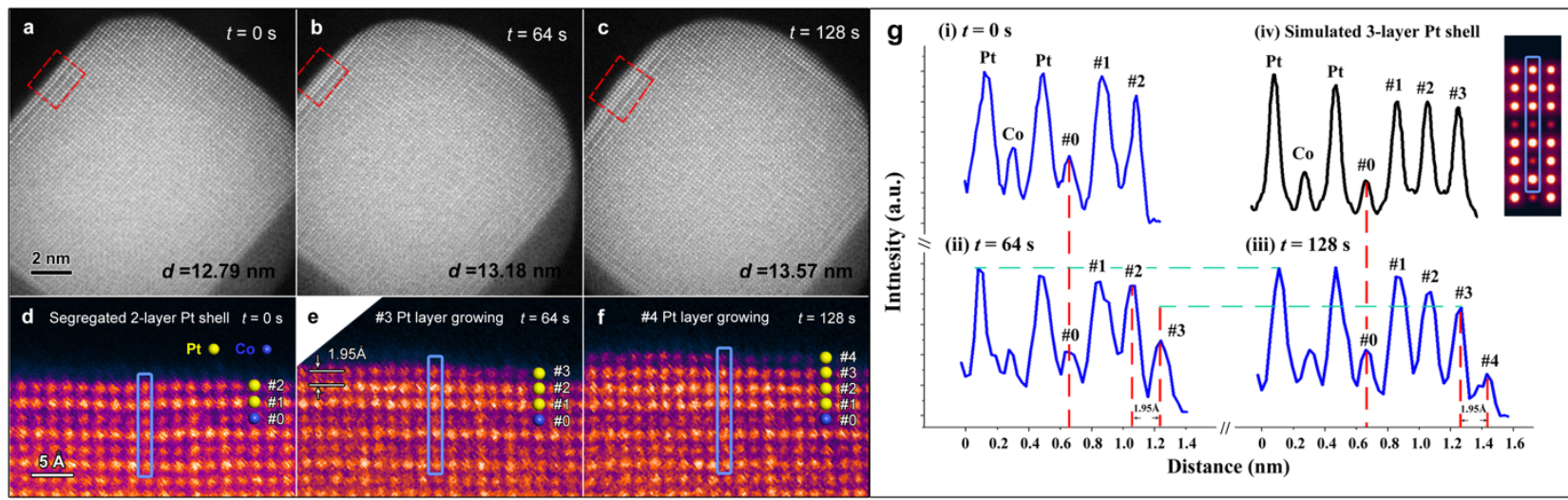

Figure 2. Layer-by-layer growth of the (100) Pt shell on $\mathrm{Pt}_{3} \mathrm{Co}$ nanoparticle during oxygen annealing. (a-c) Sequential HAADF-STEM images. (d-f) Corresponding enlarged false-colour panels of the (100) surface (indicated by red boxes) in (a-c), respectively. (g) Intensity profiles taken along the blue lines in $\mathrm{d}-\mathrm{f}$, and the simulated result, respectively. 